\title{
The effect of interleukin-2 on canine peripheral nerve sheath tumours after marginal surgical excision: a double-blind randomized study
}

\author{
Annika N Haagsman ${ }^{1 *}$, Astrid C S Witkamp ${ }^{1}$ Bart E Sjollema², Marja J L Kik³ and Jolle Kirpensteijn ${ }^{1}$
}

\begin{abstract}
Background: The objective of this study was to evaluate the effect on outcomes of intraoperative recombinant human interleukin-2 injection after surgical resection of peripheral nerve sheath tumours. In this double-blind trial, 40 patients due to undergo surgical excision ( $<5 \mathrm{~mm}$ margins) of presumed peripheral nerve sheath tumours were randomized to receive intraoperative injection of interleukin-2 or placebo into the wound bed.
\end{abstract}

Results: There were no significant differences in any variable investigated or in median survival between the two groups. The median recurrence free interval was 874 days (range 48-2141 days), The recurrence-free interval and overall survival time were significantly longer in dogs that undergone the primary surgery by a specialist-certified surgeon compared to a referring veterinarian regardless of whether additional adjunct therapy was given.

Conclusion: Overall, marginal excision of peripheral nerve sheath tumours in dogs resulted in a long survival time, but adjuvant treatment with recombinant human interleukin-2 (rhlL-2) did not provide a survival advantage.

Keywords: Sarcoma, Dog, rhIL2, PNST, Surgery, Intralesional, Injection

\section{Background}

Peripheral nerve sheath tumours (PNSTs) are spindle cell tumours that arise from the connective tissue components of the peripheral nerve and which can infiltrate the subcutis locally [1]. These tumours are thought to arise from perineural fibroblasts, which produce the non-myelinated connective tissues that surround the myelinated nerve fibre [2-6]. PNSTs appear to be pseudo-encapsulated, are locally invasive, and grow relatively slowly. Although PNST rarely metastasize, local recurrence is described commonly after resection [7-11].

Surgical removal of PNST with margins of minimally 2-3 cm margins in all dimensions is the therapy of choice. Marginal excision, which is defined as resection through the tumour pseudocapsule or surrounding reactive tissue, increases the likelihood of local recurrence and necessitates re-excision or postoperative radiation [12]. Since surgical removal alone does not guarantee

\footnotetext{
* Correspondence: a.n.haagsman@uu.nl

'Department of Clinical Sciences of Companion Animals, Faculty of Veterinary Medicine, Utrecht University, Postbus 80154, 3508, TD Utrecht, The Netherlands

Full list of author information is available at the end of the article
}

complete eradication of the tumour, radiotherapy is advocated as adjuvant therapy, and especially when tumours are marginally or incompletely excised, to prolong survival and to decrease the incidence of recurrences. Radiotherapy can also be given preoperatively to decrease the size of the tumour $[7,13]$. Chemotherapy has also been used as adjuvant therapy for canine PNST with mixed results [7,13-15].

Immunotherapy has become popular in human medicine and decreases the rate of recurrence and metastases in a variety of tumours, including sarcomas [16-18]. Systemically administered cytokines, such as interleukin-2 (IL-2), activate the immune system to enhance immunemediated responses against the tumour, thereby diminishing its metastatic potential [19]. IL-2 is a T-cell growth factor and induces clonal expansion of antigen-specific $\mathrm{T}$ cells and activation of antigen-presenting cells (APC). It also increases the production of cytokines, such as interferon- gamma (IFN- $\gamma$ ), tumour necrosis factor alpha (TNF- $\alpha$ ), and interleukin-6 (IL-6), and stimulates natural (NK) and lymphokine-activated killer cells (LAK) [11]. IL2 modulates or promotes major histocompatibility complex (MHC) antigen expression, driving the tumour

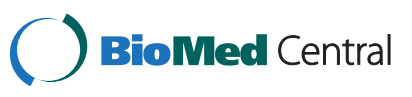


towards regression. In human cancers [20], recombinant human IL-2 (rhIL2) is commonly used as adjuvant to chemotherapy or radiotherapy [16,21], and its intratumoural administration has proven beneficial in melanoma and nasopharyngeal carcinoma, providing a complete response rate of $62.5 \%$ and a partial response rate of $21 \%$; the rate of progressive disease was $16.5 \%$ [22].

Local rhIL2 therapy (intratumoural, peritumoural, or intravesical) is suggested to be more effective than systemic rhIL2 therapy and to have fewer side effects $[17,20,23]$. It has proven effective in the treatment of soft tissue sarcomas (STS) [22,23], malignant lymphoma [24], and gastrointestinal tumours [25] in humans, mice, and dogs. Local injection of rhIL2 leads to extravasation of erythrocytes at the injection site [26], causing stagnation of blood flow and leading to tumour cell death. At a later stage, leukocytes migrate to the dead tumour cells, forming a granuloma and causing angiogenesis [20]. Locally applied rhIL2 also decreases the size of distant metastases by an as yet unknown mechanism [24,26-28]. Local IL-2 therapy has been studied extensively in mice [29-32]. A study has reported its use in dogs with confirmed STSs, where treatment consisted of Staphylococcus aureus enterotoxin A and canine interleukin-2 (L_SEA/cIL-2) [33]. The treatment was well tolerated and had antitumour activity. Other tumours successfully treated with intratumoural rhIL2 therapy include canine transmissible venereal tumours [34], bovine ocular squamous cell carcinomas [35], equine sarcoid tumours [36], and canine mast cell tumours [37].

To the authors' knowledge, the effect of adjuvant intralesional rhIL2 after surgical resection has not been studied in dogs. If rhIL2 has local or systemic effects, it should decrease the rates of recurrence and distant spread. The aim of this study was to evaluate the possible beneficial effect of local rhIL2 administration after marginal PNST removal in dogs.

\section{Methods}

In the period between 2000 and 2003, 40 dogs (any breed, age, or sex) with a clinical diagnosis of PNST were referred to two referral practices in the Netherlands (Department of Clinical Sciences of Companion Animals, Faculty of Veterinary Medicine, Utrecht University [UU] and Animal Medical Centre Amsterdam [AMCA]). The inclusion criterion was presumed PNST that could not be removed with adequate $3-\mathrm{cm}$ margins. This study included 21 dogs without previous surgery and 19 dogs with previous surgery. All the cases that were presented with previous surgery had macroscopic recurrences. Dogs with metastases or multiple tumours were excluded. The following data were collected: sex, age, weight, PNST localization (1. phalanges to carpus, 2 carpus to elbow, 3 . elbow to shoulder, 4. cervical region, 5. phalanges to tarsus, 6. tarsus to stifle, 7. stifle to hip, 8. flank and 9. head), fine-needle biopsy and histology findings, location of metastasis and tumour grade. All dogs were screened for metastases, using right lateral, left lateral, dorsoventral or ventrodorsal radiographs of the lungs. A fine-needle aspiration biopsy (FNAB) was taken from all tumours. All tumours were removed with margins of $5 \mathrm{~mm}$ or less and submitted for histological examination by a certified pathologist (MK) to determine the type and grade of the tumour and the completeness of resection.

Recombinant human IL-2 (rhIL2; specific activity $18 \times$ $10^{6} \mathrm{IU} / \mathrm{mg}$; Proleukin ${ }^{\circ}$ a gift from Chiron, Amsterdam, the Netherlands) was reconstituted to $1 \mathrm{mg} / \mathrm{ml}$ with distilled water. Polygeline was added to increase the stability of interleukin. Further dilutions were made with phosphate-buffered saline (PBS) supplemented with $0.1 \%$ bovine serum albumin, fraction V (BSA; Sigma Chemical Co., MO USA) [38]. The placebo contained distilled water with polygeline. Directly after surgical removal of the tumour, rhIL2 (1 ml containing 4.5 million IU) or placebo was injected evenly into the wound bed (0.01$0.05 \mathrm{ml}$ per site) in an at random double-blinded fashion $[20,34]$. The wound bed was larger than the tumour by $5 \mathrm{~mm}$ in all dimensions and depended on the tension in that specific area. The exact wound size was not measured before or after injection. Surgery was performed by one of two surgeons (BS and JK). None of the patients received postoperative radiotherapy or chemotherapy. Blinding was broken after data had been accumulated and analysed.

Directly after surgery, the wounds were evaluated for signs of redness, haemorrhage, swelling, tenderness, and possible wound closure defects. All dogs were routinely screened for physical signs of regrowth and radiographic signs of metastasis at $1,3,6$, and 12 months after surgery and every 12 months thereafter. If clinical signs developed before the screening time points, dogs were examined immediately. Recurrence was confirmed by evaluation of cytological or histological biopsy specimens. At the end point of the study, either the dog owner or the referring veterinarian was contacted by telephone for follow-up and administered a standard questionnaire including questions about breed, sex, age, disease-free interval, metastasis occurrence, tumour recurrence, survival time, and cause of death (if relevant). The investigators who administered the questionnaires were blinded to the treatment received.

Overall survival time (OS) was defined as the interval between the date of surgery and death due to the disease or the date on which the dog was last known to be alive. Metastasis free (MFI) and recurrence free (RFI) interval was defined as the interval between the date of the surgery and the date of either metastases or recurrence, or if there were no signs of recurrence or metastases, as the 
interval between surgery and the time on which the dog was last known to be alive. Dogs that had died from unrelated causes or which were still alive at the time of follow-up and without signs of either recurrence or metastases were considered censored. All dogs had a follow-up longer than 5 years. If a patient died without follow-up, the case was classified as lost to follow-up (LTF) and the last physical and diagnostic examination was used as end point. If a physical examination was performed but no diagnostic investigations, only RFI and OS were recorded; MFI was then coded as LTF. Owners were asked detailed questions about the surgical site, to determine whether RFI could be established; if there was any doubt, the date of the last physical examination was used in analyses.

\section{Statistical methods}

A power analysis was performed in advance of the study, using survival data obtained from previous studies $[8,11]$. With an expected mean RFI difference of $20 \%$ per procedure and a variation coefficient of $15 \%$, an $\alpha$ of 0.05 , and a $\beta$ of 0.15 , a total sample size of 40 dogs was calculated.

Frequency distributions were calculated and categorical data were compared using Chi-square analysis. Fisher's exact test was used when sample sizes were small, i.e. if more than $25 \%$ of the samples were smaller than 5 . Normally distributed, continuous and interval categorical data were analysed using an analysis of variance (ANOVA). Logarithmic transformation was performed on variables that were not normally distributed. A hazard ratio (HR) of different variables on MFI, RFI, and OS was calculated using multivariate Cox proportional hazards analysis. $\mathrm{P}<0.05$ was considered significant. The KaplanMeier product limit method was used to estimate median RFI and OS. Group comparisons were made using the mantel-Cox log rank test (SPSS version 20.0). Statistical significance was defined as $\mathrm{P}<0.05$.

\section{Results}

Marginal excision ( $<5 \mathrm{~mm}$ ) was the only surgical option for all tumours. The dogs were of different breeds; 4 Beagles, 3 Boxers, 4 Bouviers des Flandres, 2 Bull Terriers, 8 cross breeds, 2 Fox terriers, 3 Bernese Mountain Dogs, 4 Labrador Retrievers, and one of each following breeds: English Bulldog, German shorthaired pointer, Leonberger, Husky, Belgian Shepherd Dog, German Shepherd Dog, White Shepherd Dog, Groenendaeler, Flatcoated Retriever, and Irish Wolfhound. Most were large-breed dogs; the median weight was $31 \mathrm{~kg}$ (range 12-51 kg). There were 23 male dogs and 17 female dogs. The median age of the dogs at referral was 9 years (range 3-14). Nineteen dogs had previously undergone tumour resection.
Four tumours were located on the forelimb in the area from the phalanges until the carpus, 15 from the carpus until the elbow, 7 from the elbow until the shoulder, 5 from the tarsus until the stifle, 4 from the stifle until the hip, 4 on the head, and 1 on the flank (Table 1). Most tumours were located at the antebrachium (38\%) or the brachium (18\%). Fine-needle aspiration biopsies (FNABs) were used to diagnose 13 mesenchymal proliferations and 15 mesenchymal tumours; the FNABs of 12 dogs were not diagnostic. There were 29 neurofibrosarcomas, 4 fibrosarcomas, 2 myxosarcomas, and 5 haemangiopericytomas. Tumour grade was determined to be low (grade 1) in 8 cases, medium (grade 2) in 20 cases, and high (grade 3 ) in 12 cases. The median tumour volume was $38.5 \mathrm{~cm}^{3}$ (range $\left.1-2890 \mathrm{~cm}^{3}\right)$. Six dogs developed metastatic disease in the lungs (5 dogs) and lymph nodes $(1 \mathrm{dog})$. All tumours had dirty surgical margins (tumour reached or extended into the surgical margin).

There was no significant difference between the control group and the IL-2 group in sex, age, weight, tumour localization, left versus the right side, radiographic appearance of the tumour, results of fine-needle biopsy, location of metastasis, and tumour grade (Table 1). There were also no between-group differences after exclusion of dogs with myxosarcomas and haemangiopericytomas, which are not classified as true PNSTs. The side effects of therapy were minimal and could not be distinguished from the normal side effects observed after skin surgery, such as minor redness, haemorrhage, tenderness of the wound, and wound swelling. No wound dehiscence was observed. There was no significant between-group differences in side effects (data not shown).

\begin{tabular}{|c|c|c|c|}
\hline & rhlL2 & Placebo & Significance \\
\hline Sex & $12 \mathrm{M}: 8 \mathrm{~F}$ & $10 \mathrm{M}: 10 \mathrm{~F}$ & NS \\
\hline \multirow[t]{3}{*}{ Localisation } & Front leg 10 & Front leg 14 & NS \\
\hline & Rear leg 6 & Rear leg 3 & \\
\hline & Axial 2 & Axial 3 & \\
\hline Left vs right & L 9: R11 & L14: R6 & NS \\
\hline \multirow[t]{3}{*}{ Grade } & Grade 1: 2 & Grade 1: 6 & NS \\
\hline & Grade 2: 11 & Grade 2: 9 & \\
\hline & Grade 3: 7 & Grade 3: 5 & \\
\hline Previous sx & yes: no 10:10 & yes: no 9:11 & NS \\
\hline Size $^{*}$ & $45.6 \pm 16.0$ & $19.8 \pm \underline{4.0}$ & NS \\
\hline Age $^{*}$ & $9.3 \pm \underline{0.55}$ & $8.9 \pm \underline{0.60}$ & NS \\
\hline Weight* & $33.1 \pm 1.7$ & $29.2 \pm 2.1$ & NS \\
\hline
\end{tabular}

NS No significant difference between the two groups, vs Versus, sx Surgery, $M$ Male, $F$ Female, $L$ Left, $R$ Right, axial Head or trunk; * described as mean \pm standard error. 
Overall, the median RFI was 874 days (range 482141 days), the median MFI was 1884 days (range 4072141 days) and the median OS was not reached (range 197-2141 days). There was no significant difference in OS between the treatment groups (Figure 1) or by tumour group or grade. The rate of recurrence was $45 \%$ (9/20) in the rhIL2 group and 35\% (7/20) in the placebo group. Six dogs developed metastatic disease, 5 in the lungs and 1 in the lymph nodes; all dogs had previously undergone surgery. Metastases developed in 3 dogs (15\%) that had received placebo and in 3 dogs that had received rhIL2 and the incidence of metastases did not differ significantly between treatment groups. Of the 6 dogs with metastases, 2 were from grade $1(n=8), 3$ from grade 2 $(\mathrm{n}=20)$ and 1 from grade 3 tumors $(\mathrm{n}=12)$. There was no significant difference in incidence of metastases between the three groups.

The only significant difference in OS $(\mathrm{P}=0.006)$ was found for dogs that had previously undergone surgery compared with those that had their first surgery at the referral clinics (UU or AMCA; Figure 2). Using multivariate analysis, the hazard ratio (HR) for recurrence was higher in dogs that had previously undergone surgery at the time of inclusion compared to dogs that had not had prior surgery ( $\mathrm{p}<0.001$, HR 10.7, 95\% CI 2.321.2; Figure 3 ). The only other factor that had a significant $\mathrm{HR}$ was the weight $(\mathrm{P}=0.02, \mathrm{HR}=1.08,95 \% \mathrm{CI}$ 1.01-1.16). There was no significant difference in occurrence of metastases between dogs that had undergone previous surgery and the ones that had not, using multivariate analysis.

\section{Discussion}

This study describes the effect on surgical outcomes of local injection of rhIL2 or placebo into the wound bed after surgical excision of PNST-like tumours. Treatment with rhIL2 did not significantly influence the MFI, RFI, or OS. In contrast, it has been reported to have a beneficial effect in various types of tumours in humans $[16,17]$ and animals [25,29-31,35,36,39], providing a 6\% complete response $(\mathrm{CR})$ and $10 \%$ partial response (PR) in one study [16] or a $50 \%$ decrease in nasal angiosarcoma size in another [17]. In other studies, mice with inoculated lymphoma or mastocytoma treated intraperitoneally with IL-2 achieved a 70-90\% CR [29-31], cattle with ocular squamous cell carcinoma treated with peritumoural IL-2 achieved a 69\% CR [35], and horses with sarcoid tumours achieved a $14 \%$ PR and $43 \%$ CR with intratumoural IL-2 [36]. While systemic administration of IL-2 has also been used, locoregional administration strategies are more effective and successful $[23,26]$. Interestingly, local treatment is often effective systemically, eradicating metastases [32]. Systemic IL-2 therapy can lead to life-threatening toxicities and to hepatic dysfunction, which might result in hypotension and vascular fluid accumulation [23].

The use of IL-2 as sole adjuvant agent after sarcoma resection is not warranted at the moment. Additional therapies, such as chemotherapy or radiotherapy, may increase the efficacy of IL-2, but a large effect is unlikely, especially when a wide surgical excision is used, which is the preferred approach for PNSTs. In the abovementioned study of horses with sarcoid tumours, local IL-2 treatment combined with chemotherapy (cisplatin)

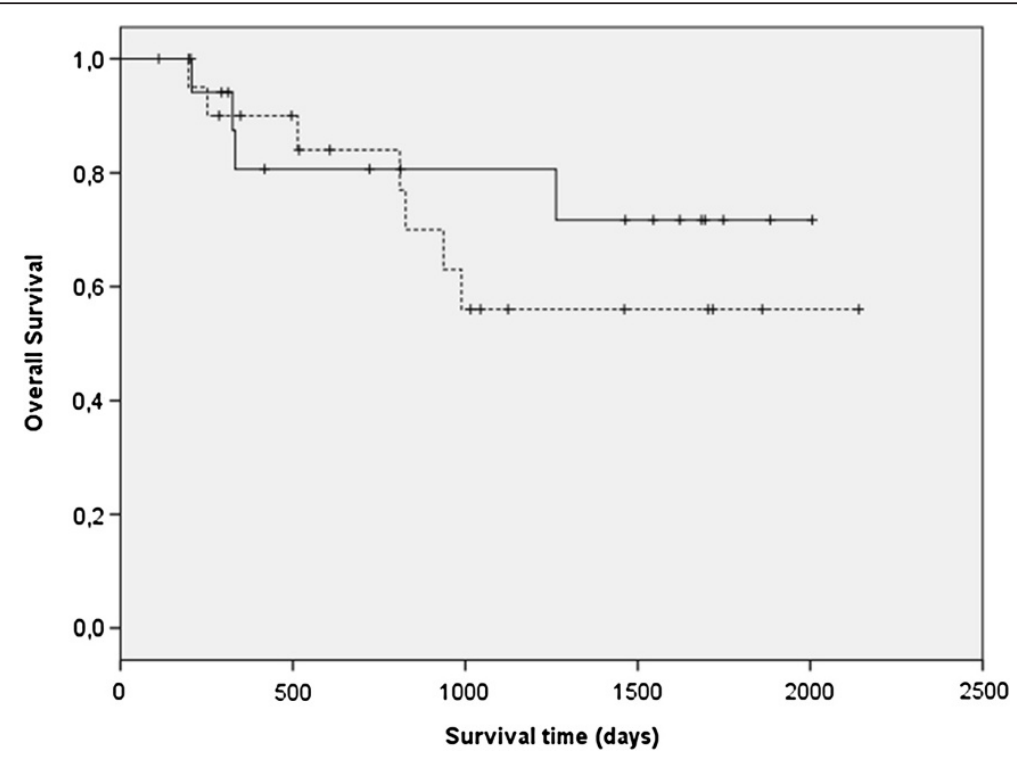

Figure 1 Kaplan-Meier survival curve of overall survival (OS) showing a non-significant difference between dogs with PNSTs treated with rhIL2 (dotted line) versus placebo (continuous line). 


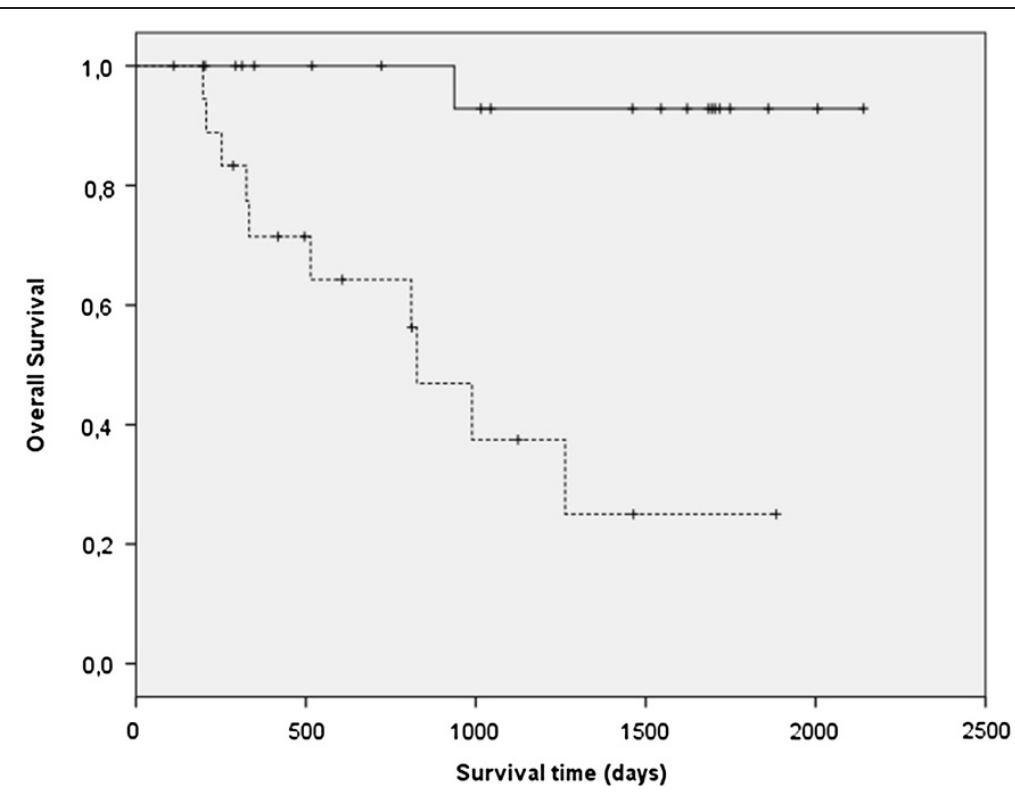

Figure 2 Kaplan-Meier survival curve of overall survival (OS) showing a significant difference $(P<0.001)$ between dogs with PNSTs that had undergone a previous surgery (dotted line) versus the ones that did not (continuous line).

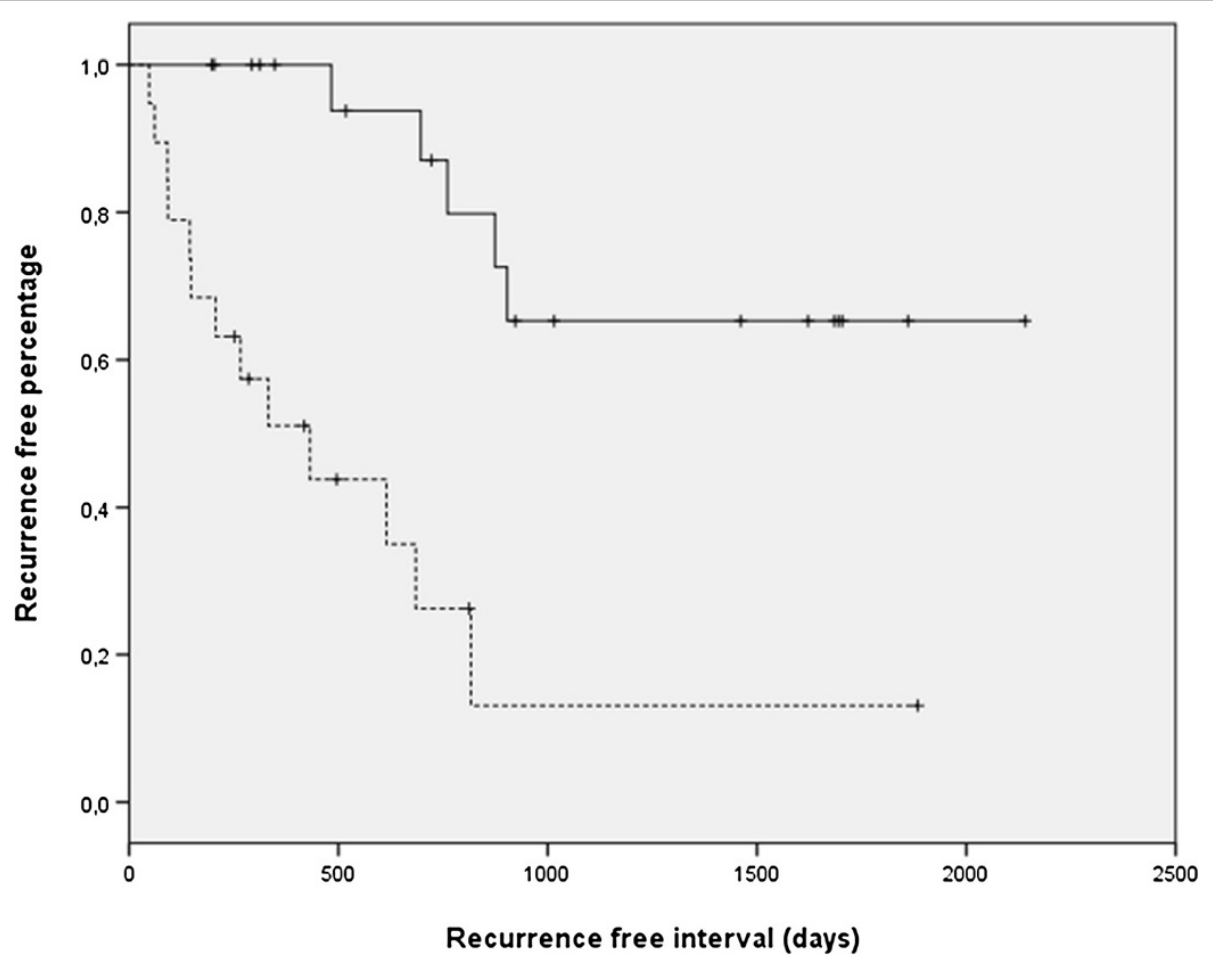

Figure 3 Kaplan-Meier survival curve of recurrence free interval (RFI) showing a significant difference $(p<0.001)$ between dogs with PNSTs that had undergone a previous surgery (dotted line) versus the ones that did not (continuous line). 
resulted in better outcomes than local IL-2 monotherapy (53\% PR and $80 \%$ CR compared with $14 \%$ PR and $43 \% \mathrm{CR}$, respectively) [36].

Incomplete tumour resection increases patient morbidity, treatment costs, risk of further recurrence, and ultimately decreases survival time [40-43]. Therefore, the likelihood of recurrence is much lower if surgery is performed by a specialist-certified surgeon rather than a referring veterinarian [13]. We found a much lower recurrence rate in dogs that had not previously undergone surgery than in dogs that had previously undergone surgery. This difference can be explained in two ways: the veterinarians who removed the primary tumour might have used even smaller tumour margins than the specialistcertified surgeon or the previously removed tumour might have been more aggressive and more likely to recur. Of the 40 dogs, 8 had a grade 1 PNST, 20 a grade 2, and 12 a grade 3 . There was no significant difference in grade of the removed PNST between the dogs with or without previous surgery. Nor did we find survival or recurrence rates to differ by tumour grade, but this might be a reflection of the relatively low number of cases.

The rate of recurrence was similar in the two treatment groups, 45\% (9/20; rhIL2 group) versus 35\% (7/20; placebo group). The overall recurrence rate (40\%) was higher than the $15 \%$ reported in a comparable study of 41 dogs, in which all dogs that had surgery for recurrence had been referred after inadequate primary tumour resection [44]. This difference might be because we had a longer follow-up of minimally 5 years. Interestingly, tumour recurred at the site of excision many years after primary surgery, suggesting that dormant cells were present. This suggests that future studies should have longer follow-up times ( $>2$ years) and that postoperative radiotherapy is warranted. Radiotherapy has been used in many studies after marginal resection of STS, with recurrence rates varying from $15 \%$ to $31 \%$. Irradiated, incompletely resected STSs had a recurrence rate of $15 \%$ [42] and 17\% [43]. However, because these studies did not investigate PNSTs and did not include rhIL2 therapy, we cannot compare results. Interestingly, a recent review of human cancer concluded that the response rate was higher when rhIL2 was used as adjuvant to radiotherapy [16], an approach that should be evaluated in the future. Alternative treatment options for incomplete tumour resection are re-excision of the wound bed with wider margins $[40,42,44]$ and downstaging local disease with preoperative radiotherapy [40].

Most PNSTs in dogs have low metastatic rates. In this study, $15 \%$ of the PNSTs (6/40) had metastasized to lungs or lymph nodes, and rhIL-2 therapy did not influence the rate of metastasis. One study of IL-2 used in an adjuvant setting as local inhalation therapy for carcinoma reported that 2 of 7 treated dogs achieved full remission for more than a year (29\%) [45]. Other canine STS studies have reported lower metastasis rates than the rate reported here. The rate of metastasis was $8 \%$ in a study in which radiotherapy was used as adjuvant therapy after incompletely resection [42]. The higher metastatic rate in our study might have been due to the longer follow-up, a more vigilant surveying system, or the presence of dormant cells in the tumour margins. The results of this study indicate that aggressive primary surgery is advisable for these tumours.

The higher incidence of metastasis may warrant the use of postoperative chemotherapy, a protocol not used in this study. However, the exact role of chemotherapy in preventing distant metastasis of STS is not known. Doxorubicin, ifosfamide, and mitoxantrone have been used in dogs and in humans. The overall response of canine sarcomas to doxorubicin was $23 \%$ in one study [46], but doxorubicin had no effect as adjuvant therapy in a recent study of high-grade STS [14]. Cisplatin applied in a biodegradable implant delivery system directly after marginal resection of STS in 19 dogs resulted improved survival in 9 dogs $(47 \%)$ with a median follow-up of 874 days; 8 dogs died of tumour-unrelated causes, and 3 dogs had a recurrence (fatal in 1 dog) [47]. Recently, cyclophosphamide combined with piroxicam significantly prolonged the DFI of 30 dogs (median DFI $>410$ days) compared with that of 55 control dogs (median DFI 211 days) after marginal resection of STSs [43]. The median OS was not reached in our study, even though follow up times reached over 5 years. This compares favourable to other studies reported for surgery alone (1416 days) [41] or 2270 days after incomplete resection combined with adjuvant radiotherapy [42] and the 309 days reported in a study of intraoperative chemotherapy [47] or fourfraction palliative radiotherapy [48]. Chemotherapy is typically used in dogs with high-grade and incompletely resected tumours with a high metastatic rate, which might explain the high recurrence rate [13].

PNSTs on extremities often cannot be excised with wide margins and amputation of the limb is a suggested treatment alternative. However, marginal resection either as sole therapy or combined with radiotherapy may result in a similar long-term survival with less immediate morbidity. Marginal excision of low-grade $\left(G_{1}\right)$ STSs from the extremities of 35 dogs resulted in only 4 recurrences (11\%) [49]. Similarly, a study showed a gradedependent recurrence after marginal excision in 7\% (3/ $41)$ of grade $1\left(G_{1}\right)$ tumours, $34 \%(14 / 41)$ of grade $2\left(G_{2}\right)$ tumours, and $75 \%(3 / 4)$ of grade $3\left(\mathrm{G}_{3}\right)$ tumours [41]. Survival time was not influenced by grade in dogs treated with marginal excision [50]. Radical resection of STSs on extremities by limb amputation should therefore be considered as a last resort for recurrent and high-grade tumours, taking into account that the risk of metastases is 
higher in cases that had a recurrence [49,50]. A more aggressive approach, with preoperative incisional biopsies, seems warranted. Surgical margins can be smaller with lower grade tumours and still result in positive outcomes. The study reported here, had longer a follow up and a grade variation between groups that was identical but the comparison with historic data prevents any major conclusions when survival outcome is compared to the abovementioned studies.

The study had several limitations. Although the entry criteria were strict and the double-blinded study had a well-executed follow-up regimen, a major limitation remains the compliance of owners. Unfortunately, owner compliance is never $100 \%$ and some owners lived too far away to bring their dogs to follow-up evaluations. In these cases, we consulted the referring veterinarian for follow-up information, but we have no way of knowing whether he/she had recently examined the patient. The variation in tumour size, location, and grade can also affect outcomes, especially in small case cohorts. In total, 4.5 million IU of rhIL2 was administered, but the area of distribution varied with the wound size, which limits extrapolation of the exact local dose of rhIL2. The size of the wound should have been measured prior to injection of rhIL2 or placebo to allow a better calculation of the exact dose per square $\mathrm{cm}^{2}$. Power analysis indicated that 40 dogs would be needed to detect a statistically significant difference between treatments. However, this is still a small number, especially when multiple observations and variables are used, such as the grade and histologic diagnosis. The use of two instead of one referral institute may have influenced the data although there were no significant differences between the two institutes for the variables examined (data not shown).

\section{Conclusion}

The long-term prognosis of PNSTs was generally good, even after marginal resection. Wide resection of these tumours will most likely result in a better long-term RFI and OS for more malignant tumours, whereas marginal excision results in an increased recurrence rate and possible risk of metastases. This study showed that recurrences can occur years after the primary surgery, that recurrences are much more common after prior surgery, that metastases can occur in both lymph nodes and lungs, and that intralesional IL-2 does not confer a survival advantage in dogs with PNSTs. Future studies should use a stringent follow-up evaluation schedule as used in this study with minimally a 5-year follow-up to allow proper analysis of survival statistics. Although marginal excision of PNST (with or without adjuvant treatment with rhIL2) resulted in a long survival in a subset of dogs, presurgical incisional biopsy, wide excision, and/or adjuvant radiotherapy are advisable to prevent recurrence and possible metastases.

\section{Competing interests}

The authors declare that they have no competing interests.

\section{Authors' contributions}

JK and BES were responsible for collecting patients and performed the surgeries. ANH and performed the analysis of the patients documents. MJLK performed the interpretation of the histology. ANH, JK and ACSW helped to draft the manuscript. All authors read and approved the final manuscript.

\section{Acknowledgements}

We thank Prof. dr. Willem den Otter of the Department of Urology, Free University Medical Centre, Amsterdam, the Netherlands, for his initial help and guidance in setting up this project.

\section{Author details}

${ }^{1}$ Department of Clinical Sciences of Companion Animals, Faculty of Veterinary Medicine, Utrecht University, Postbus 80154, 3508, TD Utrecht, The Netherlands. ${ }^{2}$ Animal Medical Centre Amsterdam (AMCA), Isolatorweg 45, 1014AS Amsterdam, The Netherlands. 'Department of Pathobiology, Pathology, Faculty of Veterinary Medicine, Utrecht University, Yalelaan1, 3584CL Utrecht, The Netherlands.

Received: 25 March 2013 Accepted: 31 July 2013

Published: 8 August 2013

\section{References}

1. Nimwegen S, Kirpensteijn J: Specific disorders. In Veterinary surgery: small animal. 1st edition. Edited by Tobias KM, Johnston SA. USA: Saunders Elsevier; 2012:1316

2. Mauldin GN: Soft tissue sarcomas. Vet Clin Small Anim Pract 1997, 27(1):139-148.

3. Hendrick MJ, Mahaffey EA, Moore FM, et al: World Health Organization International histological classification of tumors of domestic animals. Second Series, Volume II, Histological classification of mesenchymal tumors of the skin and soft tissues of domestic animals. Washington, DC: Armed Forces Institute of Pathology in cooperation with the Americam Registry of Pathology and the World Health Organization Collaborating Center for Worlwide Reference on Comparative Oncology; 1998:16.

4. Goldschmidt MH, Shofer FS: Melanoma. In Skin tumors of the dog and cat. 2nd edition. Oxford: Elsevier Books; 1999:131-141.

5. Kaldrymidou H, Leontides L, Koutinas AF, Saridomichelakis MN, Karayannopoulou M: Prevalence, distribution and factors associated with the presence and the potential for malignancy of cutaneous neoplasms in 174 dogs admitted to a clinic in northern Greece. J Vet Med A Physiol Pathol Clin Med 2002, 49(2):87-91.

6. Pakhrin B, Kang MS, Bae IH, Parks MS, Jee H, You MH, Kim JH, Yoon BI, Choi YK, Kim DY: Retrospective study of canine cutaneous tumors in Korea. J Vet Sci 2007, 8(3):229-236.

7. Ehrhart N: Soft-tissue sarcomas in dogs: a review. J Am Anim Hosp Assoc 2005, 41:241-246.

8. Brehm DM, Vite $\mathrm{CH}$, Steinberg HS, Haviland J, van Winkle T: A retrospective evaluation of 51 cases of peripheral nerve sheath tumors in the dog. J Am Anim Hosp Assoc 1995, 31(4):349-359.

9. Harcourt-Brown TR, Granger N, Smith PM, Hughes K, Jeffery ND: Use of a lateral surgical approach to the femoral nerve in the management of two primary femoral nerve sheath tumours. Vet Comp Orthop Traumatol 2009, 22(3):229-23.

10. Gaitero L, Anor S, Fondevila D, Pumarola M: Canine cutaneous spindle cell tumours with features of peripheral nerve sheath tumours: a histopathological and immunohistochemical study. J Comparable Pathol 2008, 139(1):16-23.

11. Liptak JM, Forrest LJ, et al: Soft tissue sarcomas. In Withrow \& MacEwen's: small animal clinical oncology. 4th edition. Edited by Withrow SJ, Vail DM. St Louis, USA: Saunders Elsevier; 2007:211-234. 425-454.

12. McSporran KD: Histologic grade predicts recurrence for marginally excised canine subcutaneous soft tissue sarcoma. Vet Pathol 2009, 46(5):928-933. 
13. Ettinger SN: Principles of treatment for soft-tissue sarcomas in the dog. Clin Tech Small Anim Pract 2003, 18(2):118-122.

14. Selting KA, Powers BE, Thomson L, Mittleman E, Tyler JW, Lafferty MH Withrow SJ: Outcome of dogs with high-grade soft-tissue sarcomas treated with and without adjuvant doxorubicin chemotherapy: 39 cases (1996-2004). J Am Vet Med Assoc 2005, 227(9):1442-1448.

15. Rassnick KM: Medical management of soft-tissue sarcomas. Vet Clin North Am Small Anim Pract 2003, 33(3):517-531.

16. Seung SK, Curti B, Crittenden M, Urba W: Radiation and immunotherapy: renewed allies in the war on cancer. Oncoimmunology 2012, 1(9):1645-1647.

17. Fukushima K, Dejima K, Koike S, Tei G, Asano J, Ueda M, Hyuga M, Oshima W: A case of angiosarcoma of the nasal cavity successfully treated with recombinant interleukin-2. Otolanyngol Head Neck Surg 2006, 134(5):886-887.

18. Rosenberg SA: The development of new immunotherapies for the treatment of cancer using interleukin-2. A review. Ann Surg 1988, 208(2):121-135.

19. Hsiao YW, Liao KW, Chung TF, Liu CH, Hsu CD, Chu RM: Interactions of host IL- 6 and IFN-gamma and cancer-derived TGF-beta1 on MHC molecule expression during tumor spontaneous regression. Cancer Immunol Immunother 2008, 57(7):1091-1104.

20. Den Otter W, Jacobs JJL, Battermann JJ, Hordijk GJ, Kratev Z, Moiseeva EV, Stewart RJE, Ziekman PGPM, Koten JW: Local therapy of cancer with free IL-2. Cancer Immunol Immunother 2008, 57(7):931-950.

21. Toma S, Melioli G, Palumbo R, Rosso R: Recombinant interleukin-2 and interferon-alpha-2a in pretreated advanced soft-tissue sarcomas. Int $J$ Oncol 1993, 2(6):997-1001.

22. Radny P, Caroli UM, Bauer J, Paul T, Schlegel C, Eigentler TK, Weide B, Schwarz M, Garbe C: Phase II trial of intralesional therapy with interleukin-2 in soft tissue sarcomas. Br J Cancer 2003, 89(9):1620-1626.

23. Shaker MA, Younes HM: Interleukin-2: evaluation of routes of administration and current delivery systems in cancer therapy. $J$ Pharm Sci 2009, 98(7):2268-2298.

24. Maas RA, Dullens HF, De Jong WH, Den Otter W: Immunotherapy of mice with large burden of disseminated lymphoma with low dose interleukin2. Cancer Res 1989, 49(24 Pt 1):7037-7040.

25. Krastev Z, Koltschakov V, Tomova R, Deredjian S, Alexiev A, Popov D, Tomov B, Koten JW, Jacobs J, Den Otter W: Locoregional IL-2 low dose applications for gastro-intestinal tumors. World J Gastroenterol 2005, 11(35):5525-5529.

26. Jacobs JJ, Sparendam D, Den Otter W: Local interleukin 2 therapy is most effective against cancer when injected intratumourally. Cancer Immunol Immunother 2005, 54(7):647-654.

27. Van Es RJ, Baselmans AH, Koten JW, Van Dijk JE, Koole R, Den Otter W: Perilesional IL-2 treatment of a VX2 head-and-neck cancer model can induce a systemic anti-tumor activity. Anticancer Res 2000, 20(6B):4163-4170.

28. Bernsen MR, Van Der Velden AW, Everse LA, Dullens HFJ, Den Otter W, Heintz PM: Interleukin-2: hope in cases of cisplatin-resistant tumours. Cancer Immunol Immunother 1998, 46(1):41-47.

29. Maas RA, Henk D, Van Weering J, Dullens HFJ, Den Otter W: Intratumoral low-dose interleukin-2 induces rejection of distant solid tumor. Cancer Immunol Immunother 1991, 33(6):389-394.

30. Maas RA, Roest PA, Becker MJ, Weimar IS, Dullens HF, Den Otter W: Effector cells of low-dose IL-2 immunotherapy in tumor bearing mice: tumor cell killing by CD8+ cytotoxic T lymphocytes and macrophages. Immunobiology 1992, 186(3-4):214-229.

31. Maas RA, Becker MJ, Weimar IS, De Nooy JC, Dullens HF, Den Otter W: Transfer of tumor immunity by both CD4+ and CD8+ tumor infiltrating T lymphocytes activated in vivo by IL-2 therapy of tumor bearing mice. Immunobiology 1993, 188(3):281-292.

32. Bernsen MR, Tang JW, Everse LA, Koten JW, Den Otter WD: Interleukin 2 (IL-2) therapy: potential advantages of locoregional versus systemic administration. Cancer Treat Rev 1999, 25(2):73-82

33. Thamm DH, Kurzman ID, Macewen EG, Feinmehl R, Towell TL, Longhofer SL, Johnson CM, Geoly FJ, Stinchcomb DT: Intralesional lipid-complexed cytokine/superantigen immunogene therapy for spontaneous canine tumors. Cancer Immunol Immunother 2003, 52(8):473-480.

34. Den Otter W, Cadée J, Gavhumende R, De Groot CJ, Hennink WE, Stewart R: Effective cancer therapy with a single injection. Cancer Immunol Immunother 1999, 48:419-420.
35. Stewart RJE, Hill FWG, Masztalerz A, Jacobs JJL, Koten JW, Den Otter W: Treatment of ocular squamous cell carcinoma in cattle with interleukin-2. Vet Res 2006, 159(20):668-672.

36. Spoormakers TJ, Klein WR, Jacobs JJ, Van Den Ingh TS, Koten JW, Den Otter W: Comparison of the efficacy of local treatment of equine sarcoids with IL-2 or cisplatin/IL-2. Cancer Immunol Immunother 2003, 52(3):179-184.

37. Ziekman PGPM, Den Otter W, Tan JFV, Teske E, Kirpensteijn J, Koten J, Javobs JJL: Intratumoural interleukin-2 therapy can induce regression of non-resectable mastocytoma in dogs. Anticancer Res 2013, 33:161-166.

38. Radny P, Caroli UM, Paul T, Schlegel C, Eigentler TK, Weide B, Schwarz M, Garbe C: Phase II trial of intralesional therapy with interleukin-2 in softtissue melanoma metastases. Br J Cancer 2003, 89:1620-1626.

39. Hill FW, Klein WR, Hoyer MJ, Rutten VP, Kock JW, Steerenberg PA, Ruitenberg EJ, Den Otter W: Antitumor effect of locally injected low doses of recombinant human interleukin-2 in bovine vulval papilloma and carcinoma. Vet Immunol Immunopathol 1994, 41(1-2):19-29.

40. Dernell WS, Straw RC, Cooper MF, Powers BE, LaRue SM, Withrow SJ: Multilobular osteochondrosarcoma in 39 dogs: 1979-1993. J Am Hosp Assoc 1998, 34(1):11-18.

41. Kuntz CA, Dernell WS, Powers BE, Devitt C, Straw RC, Withrow SJ: Prognostic factors for surgical treatment of soft-tissue sarcomas in dogs: 75 cases (1986-1996). J Am Vet Med Assoc 1997, 211(9):1147-1151.

42. McKnight JA, Mauldin GN, McEntee MC, Meleo KA, Patnaik AK: Radiation treatment for incompletely resected soft-tissue sarcomas in dogs. J Am Vet Med Assoc 2000, 217(2):205-210.

43. Elmslie RE, Glawe P, Dow SW: Metronomic therapy with cyclophosphamide and piroxicam effectively delays tumor recurrence in dogs with incompletely resected soft tissue sarcomas. J Vet Intern Med 2008, 2(6):1373-1379.

44. Bacon NJ, Dernell WS, Ehrhart N, Powers BE, Withrow SJ: Evaluation of primary re-excision after recent inadequate resection of soft tissue sarcomas in dogs: 41 cases (1999-2004). J Am Vet Med Assoc 2007, 230(4):548-554

45. Khanna C, Anderson PM, Hasz DE, Katsanis MD, Neville M, Klausner JS: Interleukin-2 liposome inhalation therapy is safe and effective for dogs with spontaneous pulmonary metastases. Am Cancer Soc 1997, 79(April 1):1409-1421.

46. Ogilvie GK, Reynolds HA, Richardson RC, Withrow SJ, Norris AM, Henderson RA, Klausner JS, Fowler JD, McCaw D: Phase II evaluation of doxorubicin for treatment of various canine neoplasms. J Am Vet Med Assoc 1989, 195(11):1580-1583.

47. Havlicek M, Straw RS, Langova V, Dernell WS: Intra-operative cisplatin for the treatment of canine extremity soft tissue sarcomas. Vet Comp Oncol 2009, 7(2):122-129.

48. Lawrence J, Forrest L, Adams W, Vail D, Thamm D: Four-fraction radiation therapy for macroscopic soft tissue sarcomas in 16 dogs. J Am Anim Hosp Assoc 2008, 44(3):100-108.

49. Stefanello D, Morello E, Roccabianca P, Lussich S, Nassuato C, Martano M, Squassino C, Avallone G, Romussi S, Buracco P: Marginal excision of lowgrade spindle cell sarcoma of canine extremities: 35 dogs (1996-2006). Vet Surg 2008, 37(5):461-465.

50. Mukaratirwa S, Chipunza J, Chitanga S, Chimonyo M, Bhebhe E: Canine cutaneous neoplasms: prevalence and influence of age, sex and site on the presence and potential malignancy of cutaneous neoplasms in dogs from Zimbabwe. J S Afr Vet Assoc 2005, 76(2):59-62.

doi:10.1186/1746-6148-9-155

Cite this article as: Haagsman et al:: The effect of interleukin-2 on canine peripheral nerve sheath tumours after marginal surgical excision: a double-blind randomized study. BMC Veterinary Research 2013 9:155. 